\title{
JNK-Mediated FOXO Expression Plays a Critical Role in EGFR Tyrosine Kinase Inhibitor-Induced BIM Expression and Apoptosis
}

\author{
Kenji Takeuchi ${ }^{*}$, Anh Ho Viet ${ }^{1}$, Katsumi Kawasaki ${ }^{1}$, Kazuto Nishio ${ }^{2}$, Fumiaki Ito ${ }^{1}$ \\ ${ }^{1}$ Department of Biochemistry, Faculty of Pharmaceutical Sciences, Setsunan University, Osaka, Japan; ${ }^{2}$ Department of Genome Bi- \\ ology, Kinki University School of Medicine, Osaka, Japan. \\ Email: ${ }^{*}$ takeuchi@pharm.setsunan.ac.jp
}

Received June $26^{\text {th }}$, 2012; revised July 25 ${ }^{\text {th }}, 2012$; accepted September $16^{\text {th }}, 2012$

\begin{abstract}
BIM, a key pro-apoptotic member of the BCL-2 family of proteins, is essential for apoptosis triggered by tyrosine kinase inhibitors (TKIs) of the epidermal growth factor receptor (EGFR). However, the precise molecular mechanism by which EGFR-TKIs induce BIM expression has remained unclear. A previous study of ours showed that the activetion of c-Jun $\mathrm{NH}_{2}$-terminal kinase (JNK) is critical for the TKI-induced apoptosis in PC-9 cells, a gefitinib-sensitive human NSCLC cell line. In this study, we therefore examined the effect of JNK activation on BIM expression and further investigated the mechanism responsible for TKI-induced apoptosis in PC-9 cells. Northern blotting analysis revealed that the TKI AG1478 induced a substantial increase in the level of BIM mRNA. However, this TKI-induced increase was not observed in dominant-negative JNK overexpressing cell line J12A5 or in the TKI-resistant cell line HP-5R, in which JNK is not activated in response to AG1478. Therefore, JNK activation was correlated with the up-regulation of BIM expression. BIM is known to be a downstream target of forkhead box protein O (FOXO) transcription factors. Immunoblot analysis indicated that the levels of FOXO1, FOXO3a, and FOXO4 transcription factors increased after AG1478 treatment of PC-9 cells but that they were not increased in either J12A5 or HP-5R cells, indicating that FOXO was increased in PC-9 cells through JNK activation. FOXO1 knockdown in PC-9 cells decreased EGFR-TKI-induced BIM expression and apoptosis. These findings provide evidence that JNK activation and subsequent increased FOXO expression play a critical role in EGFR-TKI-induced BIM expression and apoptosis.
\end{abstract}

Keywords: EGFR-TKI; FOXO; BIM; JNK; NSCLC

\section{Introduction}

Enhanced expression or function of epidermal growth factor receptor (EGFR) has been documented in a variety of tumors, including non-small-cell lung cancer (NSCLC), breast cancer, and gliomas [1-4]. These alterations can occur due to increased receptor gene transcription or amplification or receptor mutations resulting in constitutive activation of the receptor tyrosine kinase $[5,6]$. Therefore, a variety of approaches to block the EGFR and its downstream signaling pathways are undergoing clinical evaluation, including the use of anti-EGFR monoclonal antibodies and small-molecule tyrosine kinase inhibitors (TKIs) $[7,8]$.

Gefitinib and erlotinib potently inhibit the tyrosine kinase activity of EGFR with minimal inhibitory activity against other tyrosine kinases. Both EGFR-TKIs competitively block the binding of ATP to the tyrosine kinase

\footnotetext{
"Corresponding author.
}

domain of EGFR and are effective therapeutic agents against NSCLC [7-9]. Various molecular studies have identified somatic mutations in EGFR as being a major determinant underlying the dramatic clinical responses to treatment with gefitinib and erlotinib [10-12]. About $90 \%$ of the somatic mutations found in responsive patients are either small deletions encompassing 5 amino acids 746 - 750 codons (delE746-A750) or missense mutations resulting in a substitution of leucine with arginine at codon 858 (L858R). NSCLC cells with these mutations show a variety of signaling pathways that are activated through the constitutive activation of EGFR tyrosine kinase. Inhibition of both phosphatidylinositol-3'$\mathrm{OH}$ kinase (PI3K)-AKT and extracellular signal-regulated kinase (ERK) pathways has been reported to be responsible for EGFR-TKI-induced apoptotic death in NSCLC cells [13-15].

BCL-2 family proteins are known to determine the outcome of an apoptotic process initiated by the release 
of cytochrome $c$ from the mitochondria [16]. BIM is a key pro-apoptotic member of the BCL-2 family of proteins and initiates apoptosis signaling by binding to and antagonizing the functions of the anti-apoptotic members of the BCL-2 family [17-19]. Previous studies have shown that the induction of BIM is essential for apoptosis triggered by EGFR-TKI [20-22]. BIM has multiple phosphorylation sites, and its expression can be downregulated through ERK-mediated phosphorylation, which targets it for ubiquitination and proteasome-based degradation [23]. Mitogen-activated protein kinase kinase (MEK) inhibition cause an increase in the expression and dephoshorylation of BIM in NSCLC cells, but to a lesser extent than that induced with gefitinib [21]. On the other hand, the effect of gefitinib on the PI3K-AKT pathway may not be required for BIM induction in NSCLC cells. Therefore, signaling pathways other than MEK-ERK and PI3K-AKT are involved in the maximal induction of BIM, and the precise molecular mechanism by which TKIs induce BIM expression has remained unclear.

The expression level of BIM is regulated at the transcription level as well as by post-translational modification. We previously investigated the signaling pathway by which EGFR-TKI induces apoptosis in PC-9 cells, a gefitinib-sensitive human NSCLC cell line with a mutation (delE746-A750) in their EGFR and found that the activation of c-Jun $\mathrm{NH}_{2}$-terminal kinase (JNK) induced by EGFR-TKI is critical for the TKI-induced apoptosis. We also found that JNK is activated through a decrease in the level of mitogen-activated protein kinase phosphatase-1 [MKP-1; 24]. In the present study we found that EGFR-TKI increased JNK-mediated FOXO expression, thereby stimulating BIM transcription and apoptosis.

\section{Materials and Methods}

\subsection{Materials}

EGF (ultra-pure) from mouse submaxillary glands was purchased from Toyobo Co., Ltd. (Osaka, Japan). Fetal calf serum (FCS) came from PAA Laboratories GmbH (Pasching, Austria). ALLN (N-acetyl-Leu-Leu-Nle-CHO), phenylmethylsulfonyl fluoride (PMSF), pepstatin A, aprotinin, and leupeptin were obtained from Sigma (St Louis, MO). MG-132 (Z-Leu-Leu-Leu-al) and U0126 (1,4-diamino-2,3-dicyano-1,4-bis(2-aminophenylthio) butadiene) were obtained from Calbiochem (Darmstadt, Germany). RPMI-1640 medium was from Nissui Pharmaceutical Co., Ltd. (Tokyo, Japan). Antibodies used and their sources were as follow: Anti-Bim(C34C5) rabbit mAb, anti-Phospho-Bim (Ser69; D7E11) rabbit mAb, anti-Foxo1 rabbit mAb, anti-Foxo3a rabbit mAb, antiFoxo4 rabbit mAb, anti-Phospho-p44/42 MAPK (Erk1/2;
Thr202/Tyr204; E10) mouse mAb, from Cell Signaling Technology, Inc. (Danvers, MA); anti- $\alpha$-tubulin antibody (clone B-5-1-2) and anti-MAP kinase antibody, from Sigma; anti-OxPhos Complex IV subunit IV, mouse $\mathrm{IgG}_{2 \mathrm{a}}$, monoclonal 20E8 (anti-cytochrome oxidase subunit IV) from Life Technologies Corporation (Carlsbad, CA); anti-( $\beta$-actin)(C-11) from Santa Cruz Biotechnology, Inc. (Santa Cruz, CA); swine horseradish peroxidase (HRP)-linked anti-rabbit Ig antibody, from DAKO (Glostrup, Denmark); and sheep HRP-linked anti-mouse Ig antibody, from GE Healthcare UK Ltd. (Buckinghamshire, England).

\subsection{Cell Culture}

Human non-small-cell lung cancer cell line PC-9 was obtained from Tokyo Medical University (Tokyo, Japan). PC-9 cells were maintained in RPMI-1640 medium (containing $10 \mu \mathrm{g} / \mathrm{ml}$ gentamycin) supplemented with $5 \%$ FBS in $5 \% \mathrm{CO}_{2}$ at $37^{\circ} \mathrm{C}$ in a fully humidified atmosphere. Exponentially growing cells were used in all experiments.

\subsection{Isolation AG1478-Resistant Cells from PC-9 Cells}

PC-9 cells were sequentially treated with AG1478, namely, at $50 \mathrm{nM}$ for 17 days, $75 \mathrm{nM}$ for 3 days, and then $100 \mathrm{nM}$ for 1 day. Subsequently, the surviving cells were plated into the wells of a 96-well microplate at 0.5 cell/well and cloned as AG1478-resistant cell lines, one of which was named HP-5R. We then examined the effect of AG1478 on the viability of both parental PC-9 and HP-5R cells.

\subsection{Determination of Cell Viability}

The anti-proliferative effect of AG1478 on PC-9 cells was assessed by using a Cell Counting Kit-8 (DOJIN, Kumamoto, Japan) according to the manufacturer's instructions. The Cell Counting Kit-8 is a colorimetric method in which the intensity of the dye is proportional to the number of the viable cells. Briefly, $200 \mu \mathrm{L}$ of a suspension of PC-9 cells was seeded into each well of a 96-well plate at a density of 2000 cells/well. After 48 h, the culture medium was replaced with $100 \mu \mathrm{L}$ of AG1478 solution at various concentrations. After incubation for $48 \mathrm{~h}$ at $37^{\circ} \mathrm{C}, 5 \mu \mathrm{L}$ of WST-8 solution was added to each well; and the cells were then incubated for a further $30 \mathrm{~min}$ at $37^{\circ} \mathrm{C}$. Thereafter, the optical density was measured at $450 \mathrm{~nm}$ by using a BIO-RAD microplate reader model 550 (BIO-RAD, Hercules, CA). Each experiment was performed by using 6 replicate wells for each drug concentration and was carried out independently 3 times. 


\subsection{Preparation of Cellular Lysates and Immunoblotting}

Preparation of cellular lysates and immunoblotting were performed as described before [25]. Briefly, cells were lysed with buffer A (20 mM Tris/HCl, pH 7.4, containing $137 \mathrm{mM} \mathrm{NaCl}, 2 \mathrm{mM}$ EGTA, $5 \mathrm{mM}$ EDTA, 1\% Nonidet P-40, 1\% Triton X-100, $100 \mu \mathrm{g} / \mathrm{ml}$ PMSF, $1 \mu \mathrm{g} / \mathrm{ml}$ pepstatin A, $1 \mu \mathrm{g} / \mathrm{ml}$ p-toluenesulfonyl-L-arginine methyl ester, $2 \mu \mathrm{g} / \mathrm{ml}$ leupeptin, $1 \mathrm{mM}$ sodium orthovanadate, 50 $\mathrm{mM}$ sodium fluoride, and $30 \mathrm{mM} \mathrm{Na} \mathrm{P}_{4} \mathrm{P}_{2} \mathrm{O}_{7}$ ). The lysates were then incubated on ice for $30 \mathrm{~min}$, and the insoluble material was cleared by centrifugation. The samples were normalized for protein content and separated by SDSPAGE, after which they were transferred to an Immobilon-P membrane (Millipore, Bedford, MA) for immunoblotting with antibodies. The relative amount of BIM and FOXO1 was analyzed with Image $\mathrm{J}(\mathrm{NIH})$ software.

\section{6. siRNA Transfection for Knockdown of FOXO1}

The transfection was performed according to the manufacturer's instruction. Briefly, PC-9 cells were plated at $1.2 \times 10^{5}$ cells in $35-\mathrm{mm}$ dishes and cultured for $24 \mathrm{~h}$. The cells were then transfected with FOXO1 siRNA oligonucleotide (Stealth ${ }^{\mathrm{TM}}$ RNAi) or non-target control oligonucleotide (Stealth ${ }^{\mathrm{TM}}$ RNAi Negative Control Medium GC Duplex \#2) by using Lipofectamine RNAi MAX (Invitrogen Corp.). After $48 \mathrm{~h}$, the transfected cells were incubated or not with AG1478 for 30 min (for FOXO1) or for $3 \mathrm{~h}$ (for BIM); and their lysates were prepared and then used for the detection of FOXO1 and BIM, done by immunoblotting. Cell survival after siRNA transfection was assessed by performing the WST-8 assay.

\subsection{Northern Blot Analysis}

Northern blot analysis was performed as described before [25]. Briefly, cells were treated with 500 nM AG1478, and total RNA was obtained by use of Isogen (Nippon Gene Tokyo, Japan). Fifteen micrograms of RNA was separated electrophoretically. The RNA was transferred to a Hybond-N+ membrane (GE Healthcare). The blots were hybridized with human BIM cDNA that had been labeled with $\left[{ }^{32} \mathrm{P}\right]$ dCTP by use of a Rediprime II DNA Labeling System (GE Healthcare).

\subsection{Mitochondrial Isolation}

Mitochondria were isolated from PC-9 cells and J12A5 cells by using a Mitochondria Isolation Kit (Pierce, Rockford, IL) according to the manufacturer's instructtions. The mitochondrial fraction was subjected to pro- tein extraction with 2X Laemmli sample buffer (4\% SDS, $20 \%$ glycerol, $0.125 \mathrm{M}$ Tris/HCl $\mathrm{pH}$ 6.8), followed by centrifugation at $15,000 \times \mathrm{g}$ at room temperature for 10 min, and then assessed for protein content with a BCA Protein Assay Kit (Pierce). $20 \mu \mathrm{g}$ of proteins were subjected to electrophoresis and analyzed by immunoblotting for BIM. The equal loading and transfer were ensured by reprobing the membranes with anti-CoxIV antibody as a marker of mitochondria.

\subsection{Cytoplasmic and Nuclear Extracts}

Preparation of cytoplasmic and nuclear extracts was performed essentially as described previously [25]. Briefly, after having been washed with ice-cold PBS, cells were lysed at $4^{\circ} \mathrm{C}$ by incubating them for $10 \mathrm{~min}$ in hypotonic buffer. After centrifugation, the supernatants were collected as cytoplasmic extracts. Nuclear extracts were prepared by resuspension of the crude nuclei in high-salt buffer at $4^{\circ} \mathrm{C}$ for $30 \mathrm{~min}$, and the supernatants were then collected after centrifugation.

\section{Results}

BIM has been identified as a key apoptotic effector of gefitinib in sensitive cells with a mutation (delE746A750) in their EGFR [20-22]. In this study we used AG1478 [4-(3-chloroanilino)-6,7-dimethoxquinazoline] as an EGFR-TKI, which inhibitor has a quinazoline structure similar to that of gefitinib and erlotinib and studied the effect of AG1478 on the expression of BIM EL, the major isoform of BIM, in PC-9 cells. The levels of BIM in both total and mitochondrial fractions increased in response to AG1478 in a time-dependent manner (Figures 1(a) and (b)). We previously demonstrated that AG1478 intensively stimulates the phosphorylation of JNK and that the level of phosphorylated JNK continues to increase for at least $24 \mathrm{~h}$ [24]. So we examined whether AG1478 induced the expression of BIM through JNK activation. As shown in Figure 1, AG1478 did not induce BIM expression in the dominant-negative JNKoverexpressing cell line J12A5, which had no detectable JNK activity, indicating that activation of JNK was essential for AG1478-induced BIM expression. We also observed that gefitinib increased the expression of BIM in PC-9 cells but not in J12A5 cells (data not shown).

JNK activity in cells is controlled by mitogen-activated protein kinase phosphatase-1 (MKP-1). To confirm a role of JNK in the increased expression of BIM after AG1478 treatment, we used pcMKP-1-transfected cell lines (M1A4 and M1B2), which were isolated as clones overexpressing MKP-1. A previous study of ours showed that JNK activation in M1A4 and M1B2 cells is not observed in response to AG1478 treatment [24]. As shown in Fig- 


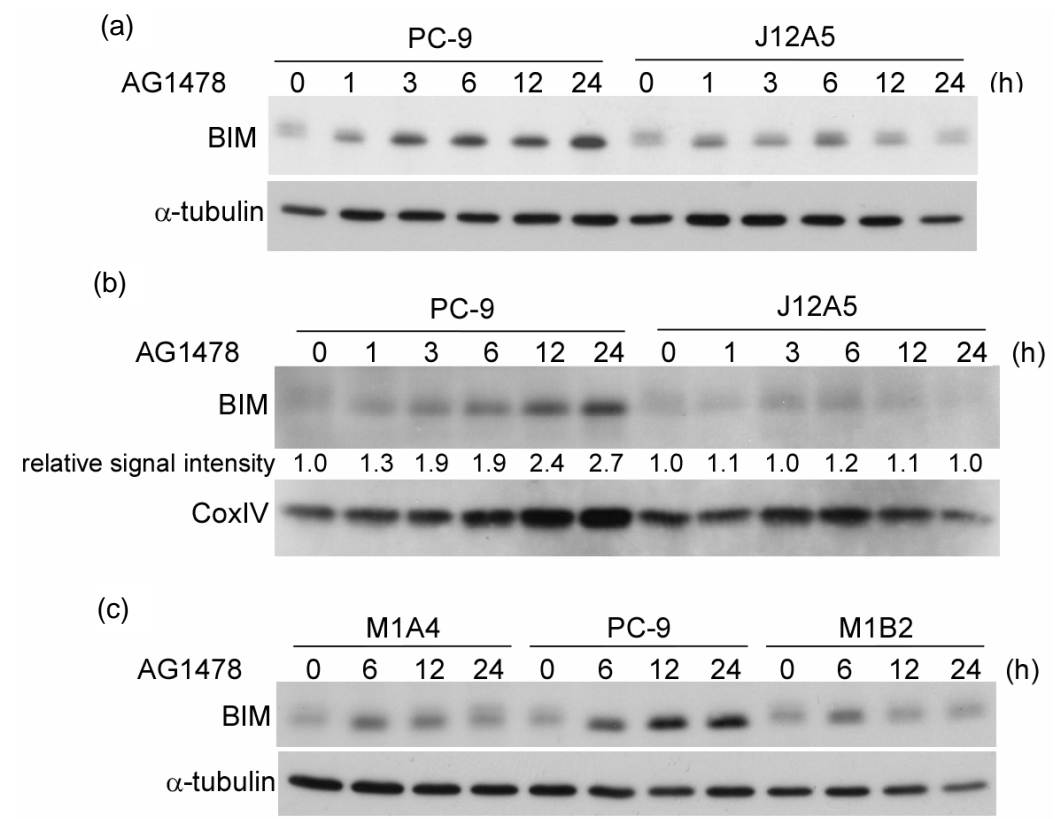

Figure 1. AG1478-induced BIM expression mediated by JNK activation. Cells were treated with 500 nM AG1478 for the indicated time periods, and extracts from the total cell (a) (c) and mitochondorial (b) fractions were prepared. The extracts were then electrophoresed on 12.5\% SDS-PAGE gels for the detection of BIM as described under "Experimental procedures". The $\alpha$-tubulin or CoxIV level was examined as a control for the equal loading of the samples. Relative signal intensity in figure B represents the ratio of the BIM signal to the CoxIV signal in each sample relative to control shown as 1. Similar results were obtained from 3 separate experiments.

ure 1(c), AG1478 did not induce BIM expression in both M1A4 and M1B2 cell lines. This result indicates that activation of JNK is essential for AG1478-induced BIM expression.

BIM is known to have multiple phosphorylation sites and its expression to be down-regulated through ERKmediated phosphorylation, which targets it for ubiquitynation and subsequent degradation in proteasomes [26, 27]. Thus, BIM expression may be regulated in PC-9 cells by multiple stimuli, including the JNK and ERK pathways. So we next determined the effect of AG1478 on BIM phosphorylation at its serine 69, which phosphorylation is known to occur in response to activation of the ERK pathway. AG1478 decreased BIM phosphorylation and concomitantly increased BIM expression (Figure 2(a)). To study the causal relationship between BIM phosphorylation and its expression level, we examined the effect of U0126, a mitogen-activated protein kinase kinase (MEK) inhibitor, on BIM expression. U0126 inhibited the phosphorylation of ERK, but its activity to increase BIM expression was low (Figure 2(b)). Since ERK-dependent phosphorylation has been reported to antagonize the proteasomal degradation of BIM, we next examined the effect of the proteasome inhibitors MG-132 and ALLN on BIM expression (Figure 2(c)). In the presence of either of these inhibitors, the BIM expression level was significantly lower than that in AG1478-treated (a)
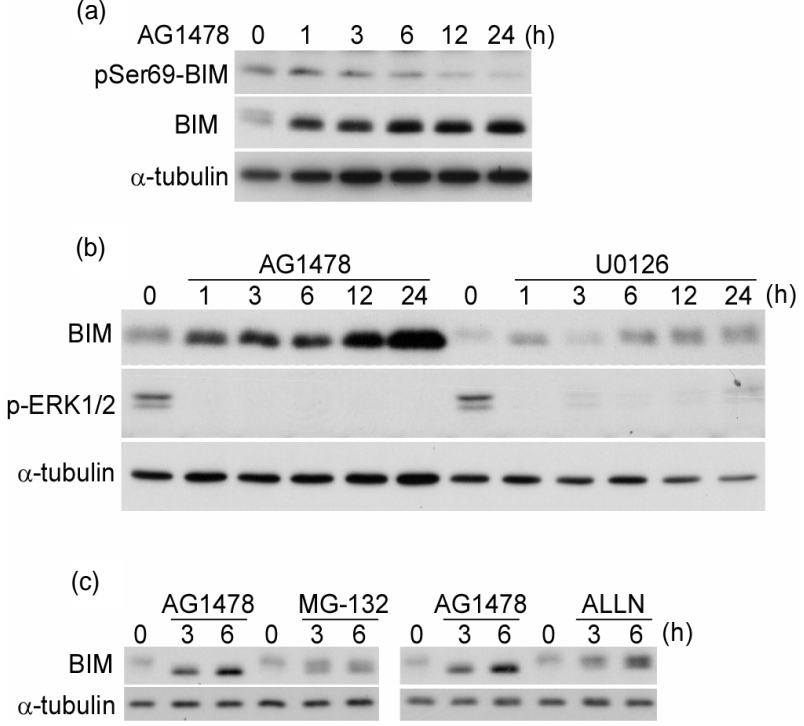

Figure 2. Effect of MEK inhibitor and proteasome inhibitors on BIM expression PC-9 cells were treated with 500 nM AG1478, $5 \mu$ M U0126 or proteasome inhibitor ALLN $(50 \mu \mathrm{M})$ or MG-132 $(1 \mu \mathrm{M})$ for the indicated time periods. The cells were then harvested, and equal aliquots of protein extracts (40 $\mu \mathrm{g}$ protein per lane) were analyzed on $12.5 \%$ SDS-PAGE gels for the detection of phospho-Ser69-BIM, BIM, and phospho-ERK1/2. The $\alpha$-tubulin level was examined as a control for the equal loading of the samples. Similar results were obtained from 3 separate experiments. 
cells (Figure 2(c)). These results indicate that AG1478 increased BIM expression mainly through JNK activation rather than by inhibition of the ERK pathway, leading to decreased phosphorylation and proteasomal degradation of BIM.

In order to investigate if the induction of BIM was regulated at the transcription step by JNK, we treated PC-9 cells with AG1478 over a time course of $3 \mathrm{~h}$, and then prepared total RNA from these cells to determine the expression of BIM mRNA by Northern blotting. AG1478 induced a substantial increase in BIM mRNA, indicating that AG1478 increased BIM transcription (Figure 3(a)). Further, no increase in BIM mRNA was observed in the dominant-negative JNK-overexpressing J12A5 cells (Figure 3(b)). It thus appears that BIM transcription was up-regulated by JNK activation in AG1478treated PC-9 cells.

Previous data from other cell systems have suggested that BIM is a downstream target of FOXO transcription factors [28-30]. To explore if FOXO could be involved in the induction of BIM expression in response to AG1478 , we carried out immunoblot analysis to study the effect of the inhibitor on the expression of FOXO proteins. The levels of FOXO1, FOXO3a, and FOXO4 transcription factors increased within $15 \mathrm{~min}$, and the FOXO1 level peaked at 30 - 60 min after AG1478 treatment (Figure 4(a)). FOXO1 expression in total and nuclear fractions was increased in response to AG1478 treatment in PC-9 cells, but they were only slightly increased in J12A5 cells (Figures 4(b) and (c)). Therefore, the level of FOXO1 was increased in the nuclei of PC-9 cells through JNK activation.

To examine whether FOXO1 was involved in the BIM expression increased by AG1478, we transfected PC-9 cells with either negative control short-interfering RNA (RNAi NC) or FOXO1 siRNAs (RNAi1 and RNAi2). As shown in Figure 5(a), an increase in the FOXO1 level was observed 30 min after the start of AG1478 treatment of PC-9 cells that had been transfected with RNAi NC, but no increase was detected in those cells transfected with FOXO1 RNAi 1 or 2 . We next studied the effect of AG1478 on BIM expression in PC-9 cells that had been transfected with FOXO1 siRNAs. A small increase in BIM expression by AG1478 was observed even in the FOXO1 knockdown PC-9 cells, but its increase was far less than that in RNAi NC-treated cells (Figure 5(b)). Our data also showed that FOXO1 knockdown in PC-9 cells resulted in decreased apoptosis in response to AG1478, as determined by cell viability (Figure 5(c)). Thus, not only FOXO1 but also other FOXO subgroup members may be involved in EGFR-TKI-induced BIM expression and cell death.

Despite the dramatic efficacy of gefitinib and erlotinib (a)

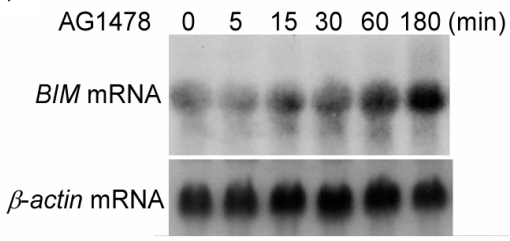

(b)

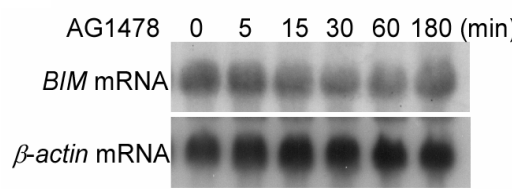

Figure 3. AG1478 increased BIM transcription through JNK pathway. PC9 (a) and J12A5 cells (b) were treated with $500 \mathrm{nM}$ AG1478 for the indicated time periods, after which Northern blot analysis of BIM mRNA was carried out as described under "Experimental procedures". The lower panel shows $\beta$-actin mRNA to demonstrate equal loading of the samples. Similar results were obtained from 3 independent experiments.

(a)

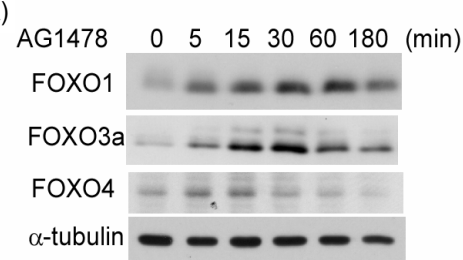

(b)

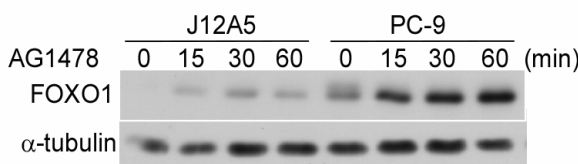

(c)

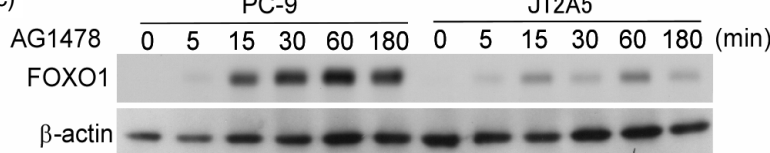

Figure 4. AG1478-induced expression of transcription factor FOXO in PC-9 cells. (a) PC-9 cells were incubated with $500 \mathrm{nM}$ AG1478 for the indicated time periods. The cells were then harvested, and equal aliquots of cell extracts (40 $\mu \mathrm{g}$ protein per lane) were examined by immunoblotting using antibodies specific for the FOXO1, FOXO3a, and FOXO4. The membrane was subsequently reprobed with an $\alpha$-tubulin antibody; (b) Cellular lysates were prepared from PC-9 and J12A5 cells. The lysates were analyzed by SDS/PAGE and immunoblotting with specific antibody against FOXO1 (upper panel) or $\alpha$-tubulin (lower panel); (c) PC-9 and J12A5 cells were treated with 500 nM AG1478 for the indicated time periods. Nuclear extracts were prepared from the cells and used for immunoblot analysis of FOXO1. The $\beta$-actin level was examined as a control for the equal loading of the samples. Similar results were obtained from 3 independent experiments. 


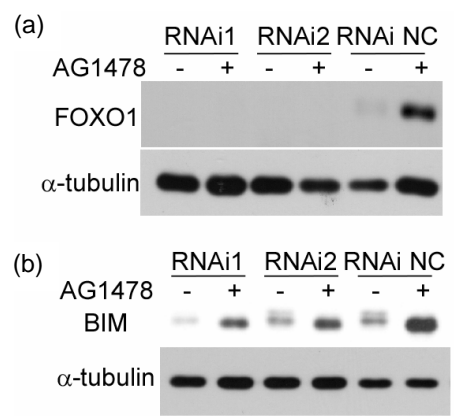

(c)

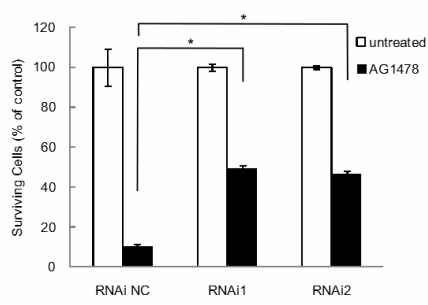

Figure 5. Transcription factor FOXO1 required for AG1478induced BIM expression and apoptosis. A and B. PC-9 cells were transiently transfected with $10 \mathrm{nM}$ FOXO1 siRNA oligonucletides (RNAi1 and RNAi2) or negative control siRNA oligonucleotides (RNAi NC). At $24 \mathrm{~h}$ post siRNA transfection, the cells were then incubated with or without $500 \mathrm{nM}$ AG1478 for an additional $30 \mathrm{~min}$ for the detection of FOXO1 (a) or for an extra $3 \mathrm{~h}$ for the detection of BIM (b). Cell lysates were examined for FOXO1 or BIM expression by immunoblotting (c). At 24 h post siRNA transfection, PC-9 cells were incubated with or without $500 \mathrm{nM}$ AG1478 for $48 \mathrm{~h}$. The viability of cells was determined by conducting WST-8 assays. The reading obtained for AG1478-untreated cells was considered as $100 \%$ viability. The data are shown as the mean \pm standard deviation (SD) $(n=$ 6). The indicated $p$-values were calculated by using the Welch two-sample t-test. ${ }^{*} p<0.01$ for the indicated comparisons. Similar results were obtained from 3 separate experiments.

for the treatment of NSCLC patients with EGFR mutations, all patients ultimately develop resistance to both of these TKIs. To analyze the mechanism responsible for this acquired resistance to TKIs, we have been isolating AG1478-resistant cell lines from PC-9 cells up to the present. So we used one of these isolated cell lines, termed HP-5R, for such analysis. As shown in Figure 6, HP-5R was resistant to AG1478 at least up to $200 \mathrm{nM}$. Since AG1478 treatment inhibited EGFR tyrosine phosphorylation in HP-5R cells, as in PC-9 cells (data not shown), no secondary EGFR mutation such as T790M or D761Y was associated with the TKI resistance of HP-5R, indicating that intracellular signaling pathways other than EGFR had changed in HP-5R cells. Interestingly, AG1478 decreased the expression of the MKP-1 protein and concomitantly stimulated the phosphorylation of JNK in PC-9 cells, but it had no effect on the expression of
MKP-1 and JNK in these HP-5R cells (Figures 6(c) and (d)). Therefore, AG1478-induced apoptosis was associated with the JNK activation. Further, AG1478 treatment did not up-regulate the expression of BIM and FOXO1 in HP-5R cells, in contrast to their up-regulation in PC-9 cells (Figures 6(e) and (f)). These results support our conclusion that JNK activation and subsequent increased FOXO expression play a role in EGFR-TKI-induced BIM expression.

\section{Discussion}

EGFR-TKIs induce apoptosis in NSCLC with EGFRactivating mutations. Recently, it was shown that BIM, one of the most potent pro-apoptotic members, is essential for apoptosis triggered by EGFR-TKIs in NSCLC with EGFR-activating mutations [20-22]. In this study, we first showed that TKI AG1478 increased the expression of BIM in NSCLC PC-9 cells, which possess one of the most common EGFR-activating mutations, i.e., deletion of exon 19. On the other hand, AG1478 did not affect the expression of other pro-apoptotic (MCL-1, $\mathrm{BAX}$, and BAD) or anti-apoptotic members (BCL-2 and BCL-xL; unpublished data).

Upon activation by growth factors, ERK phosphorylates BIM at its serine 69; and this phosphorylation targets BIM for ubiquitination and proteasome-dependent degradation [26,27]. We therefore assumed that a decrease in BIM phosphorylation by ERK would result in the accumulation of BIM in AG1478-treated PC-9 cells. Indeed, AG1478 decreased BIM phosphorylation at its serine 69 and increased BIM expression (Figure 2(a)). However, although U0126, a MEK inhibitor, inhibited the phosphorylation of ERK, it could not increase BIM expression (Figure 2(b)). Moreover, no or only a little increase in BIM expression was found in the presence of proteasome inhibitors MG-132 and ALLN (Figure 2(c)). Thus, signaling pathways other than MEK-ERK were involved to achieve maximal BIM induction, supporting a previous finding that MEK-ERK inhibition was not able to cause BIM up-regulation to the same extent seen with gefitinib [22].

The expression level of BIM is regulated not only by post-translational modification but also at the transcripttional level [31-34]. Our Northern blot analysis revealed that AG1478 induced a substantial increase in the level of BIM mRNA, indicating that AG1478 up-regulated BIM transcription in PC-9 cells. Cragg et al. previously reported that the effect of gefitinib on the PI3K-AKT pathway was not required for full BIM induction in NSCLC cells [21]. It thus appears that neither MEK-ERK nor PI3K-AKT was a major signal in TKI-induced upregulation of BIM expression. In this study, we examined whether JNK activation was associated with BIM up- 
(a)

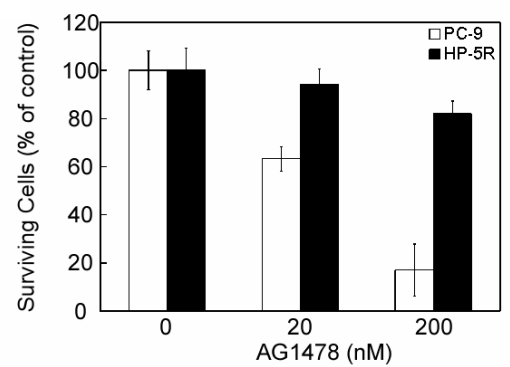

(b)

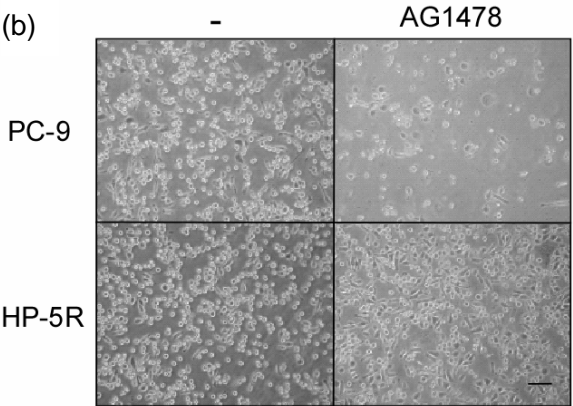

(c)

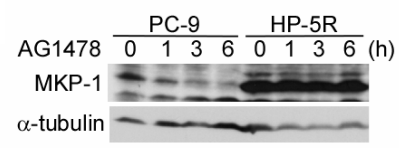

(d)

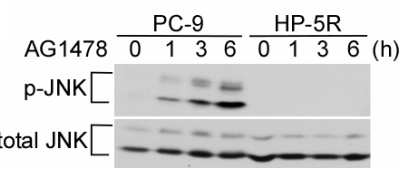

(e)

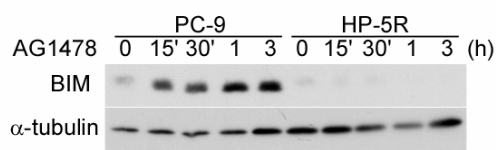

(f)

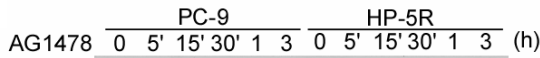

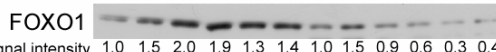
$\alpha$-tubulin - - -

Figure 6. Effects of AG1478 on the expression of MKP-1, activated JNK, BIM, and FOXO1 in AG1478-resistant cell line HP-5R. (a) PC-9 and HP-5R cells were seeded into a 96-well microplate, and treated with 20 or $200 \mathrm{nM}$ AG1478 for $48 \mathrm{~h}$. The viability of cells was determined by conducting WST-8 assays. The value of untreated cells was considered as $100 \%$ viability. The data presented are the mean \pm SD $(n=6)$; (b) PC-9 and HP-5R cells were seeded at a density of $3 \times 10^{5}$ cells per 60 -mm dish and then treated with $500 \mathrm{nM}$ AG1478. The phase-contrast photomicrographs were taken after a 48-h incubation with AG1478. Scale bar, $100 \mu \mathrm{m}$; (c) PC-9 and HP-5R cells were treated with $500 \mathrm{nM}$ AG1478 for the indicated periods of time. Total protein $(40 \mu \mathrm{g}$ protein per lane) was subjected to immunoblotting, and the membranes were hybridized with an antibody against MKP-1 (upper panel). Equal loading of the samples was checked by using an antibody against $\alpha$-tubulin (lower panel); (d) Cell lysates were analyzed on SDS-PAGE gels for phospho-JNK content (upper panel), as well as for JNK content (lower panel); (e) and (f) Cell lysates were analyzed for BIM content (e); as well as for FOXO1 content (f). The equal loading of the samples was checked by using an antibody against $\alpha$-tubulin (each lower panel). Relative signal intensity represents the ratio of the FOXO1 signal to the $\alpha$-tubulin signal in each sample relative to control shown as 1 . Similar results were obtained from 3 separate experiments.

regulation, because our previous report showed that the activation of JNK induced by EGFR-TKI was critical for the TKI-induced apoptosis [24]. Our present result showed that the TKI-induced increase in BIM mRNA was not observed in the dominant-negative JNK-over-expressing cell line J12A5. Further, the TKI-resistant cell line HP$5 \mathrm{R}$, in which JNK is not activated in response to AG1478, did not show up-regulation of BIM. Therefore, JNK activation was correlated with the up-regulation of BIM expression.

The BIM gene is a direct target of FOXO; and the 5'-UTR of the human BIM gene contains a forkheadbinding site, which is required for regulated expression of BIM following withdrawal of growth factor [23,28, 35, 36]. FOXO subfamily includes FOXO1/FKHR, FOXO3a/FKHRL1, and FOXO4/AFX [37-40]; and their activities are negatively regulated by multiple signaling pathways, such as PI3K-AKT, ERK, and IKK [41-44]. Our result showed that the levels of FOXO1, FOXO3a and FOXO4 in the PC-9 cells increased after AG1478 treatment. It thus appears that AG1478 affected FOXO expression through one or some of these multiple signaling pathways. AKT has been shown to control the activeties of FOXO through their phosphorylation, and this phosphorylation triggers their export from the nucleus and promotes binding of these transcription factors to the 14-3-3 chaperone proteins in the cytosol $[45,46]$. Indeed, our immunoblot analysis indicated that FOXO1 and FOXO3a were phosphorylated at their Akt-targeted sites (threonine 24 and serine 256 in FOXO1 and threonine 32 in FOXO3a) and that their phosphorylation level was decreased in a time-dependent manner after AG1478 treatment (unpublished data). However, the PI3K inhibittor LY294002 had little effect on the induction of BIM and FOXO in response to AG1478, although it had inhibitory activity toward the phosphorylation of FOXO at its AKT-targeted sites (unpublished data). Therefore, the PI3K-AKT-FOXO pathway was not critical for BIM induction in AG1478-treated PC-9 cells.

It has been reported that growth-factor deprivation 
causes FOXO factors to reside in the nucleus, where they are active as transcription factors, thereby resulting in pro-apoptotic signaling via the induction of TRAIL, FasL or BIM [41]. We showed that up-regulation of FOXO1 occurred in PC-9 cells after AG1478 treatment, but not in the dominant-negative JNK overexpressing J12A5 cells or in the TKI-resistant HP-5R cells, indicating that JNK activation played a role in the up-regulation of FOXO1. Recently, Wang et al. indicated that nuclear translocation of FOXO3a can be regulated by UV irradiation through a pathway from JNK through decreased ERK and Akt activities [47]. It was also earlier shown that the microtubule-targeting compound paclitaxel enhances not only the nuclear relocation of FOXO3a through JNK-dependent inhibition of the PI3K-AKT signaling pathway but also FOXO3a expression [48]. Essera et al. indicated that treatment with $\mathrm{H}_{2} \mathrm{O}_{2}$ activates Ral-mediated JNKdependent phosphorylation of FOXO4 at its threonine 447 and 451, thereby causing the nuclear translocation and transcriptional activation of FOXO4 [49]. Taken together, the data indicate it to be likely that JNK activetion was essential for the up-regulation of FOXO in PC-9 cells.

AG1478 increased BIM expression through activation of the JNK-FOXO signaling pathway, subsequently resulting in cell death. This scenario was substantiated by the knockdown experiment on FOXO1, because its knockdown led to a significant reduction in both the BIM expression and cell death (Figures 5(b) and (c)). However, FOXO1 knockdown did not completely abolish either the AG1478-induced BIM expression or the cell death. It has been reported that FOXO isoforms regulate distinct but overlapping sets of genes, indicating unique roles of FOXO isoforms in a variety of cells [50]. Thus, to define the exact role of FOXO transcription factors in PC-9 cells, a knockdown experiment on FOXO3a or double knockdown of FOXO1 and FOXO3a will be required. We previously reported that the inhibition of both survivin downregulation and BIM induction attenuate gefitinib-induced apoptosis to a greater extent than do the inhibition of either pathway alone [51]. So, both survivin downregulation and BIM induction contribute independently to gefitinib-induced apoptosis. In addition to the knockdown of FOXO isoforms, inhibition of survivin downregulation may be necessary to protect cells fully from AG1478.

In a previous paper, we showed that JNK, which is activated through a decrease in the MKP level, is critical for TKI-induced apoptosis [24]. Our present study has expanded this finding and demonstrated that JNK activetion stimulated BIM transcription through increasing FOXO1 expression. In conclusion, we propose that this MKP-JNK-FOXO-BIM pathway contributed to the in- duction of apoptosis of PC-9 cells by TKI.

Despite the dramatic efficacy of gefitinib and erlotinib in NSCLC patients with EGFR mutations, all patients ultimately develop resistance to both TKIs. A secondary mutation in EGFR (T790M) and amplification of MET have been identified as major mechanisms of acquired resistance to TKIs [52-54]. We showed herein that TKIs induced activation of MKP-JNK-FOXO-BIM pathway. Alterations in this pathway may cause resistance to gefitinib or erlotinib in NSCLC patients.

\section{Acknowledgements}

This work was supported in part by a grant-in-aid for scientific research from the Ministry of Education, Culture, Sports, Science, and Technology of Japan (\#20590077 and \#23590098). We thank Mr. T. Shinya, Mr. T. Imoto, Miss A. Nagai, and Miss S. Arasaki for their technical assistance.

\section{REFERENCES}

[1] D. S. Salomon, R. Brandt, F. Ciardiello and N. Normanno, "Epidermal Growth Factor-Related Peptides and Their Receptors in Human Malignancies," Critical Reviews in Oncology/Hematology, Vol. 19, No. 3, 1995, pp. 183232. doi:10.1016/1040-8428(94)00144-I

[2] S. B. Fox, K. Smith, J. Hollyer, M. Greenall, D. Hastrich and A. L. Harris, "The Epidermal Growth Factor Receptor as a Prognostic Marker: Results of 370 Patients and Review of 3009 Patients," Breast Cancer Research and Treatment, Vol. 29, No. 1, 1994, pp. 41-49. doi:10.1007/BF00666180

[3] T. A. Libermann, H. R. Nusbaum, N. Razon, R. Kris, I. Lax, H. Soreq, N. Whittle, M. D. Waterfield, A. Ullrich and J. Schlessinger, “Amplification, Enhanced Expression and Possible Rearrangement of EGF Receptor Gene in Primary Human Brain Tumours of Glial Origin,” Nature, Vol. 313, No. 5998, 1985, pp. 144-147. doi:10.1038/313144a

[4] A. J. Wong, S. H. Bigner, D. D. Bigner, K. W. Kinzler, S. R. Hamilton and B. Vogelstein, "Increased Expression of the Epidermal Growth Factor Receptor Gene in Malignant Gliomas Is Invariably Associated with Gene Amplification," Proceedings of the National Academy of Sciences of the United States of America, Vol. 84, No. 19, 1987, pp. 6899-6903. doi:10.1073/pnas.84.19.6899

[5] A. W. Burgess, H. S. Cho, C. Eigenbrot, K. M. Ferguson, T. P. Garrett, D. J. Leahy, M. A. Lemmon, M. X. Sliwkowski, C. W. Ward and S. Yokoyama, "An Openand-Shut Case? Recent Insights into the Activation of EGF/ErbB Receptors,” Molecular Cell, Vol. 12, No. 3, 2003, pp. 541-552. doi:10.1016/S1097-2765(03)00350-2

[6] A. Citri and Y. Yarden, "EGF-ERBB Signalling: Towards the Systems Level," Nature Reviews Molecular Cell Biology, Vol. 7, No. 7, 2006, pp. 505-516. doi:10.1038/nrm1962 
[7] R. S. Herbst and P. A. Bunn Jr., "Targeting the Epidermal Growth Factor Receptor in Non-Small Cell Lung Cancer," Clinical Cancer Research, Vol. 9, No. 16, 2003, pp. 5813-5824.

[8] A. F. Gazdar, H. Shigematsu, J. Herz and J. D. Minna, "Mutations and Addiction to EGFR: The Achilles 'Heal' of Lung Cancers?” Trends in Molecular Medicine, Vol. 10, No. 10, 2004, pp. 481-486. doi:10.1016/j.molmed.2004.08.008

[9] K. Nakagawa, T. Tamura, S. Negoro, S. Kudoh, N. Yamamoto, N. Yamamoto, K. Takeda, H. Swaisland, I. Nakatani, M. Hirose, R. P. Dong and M. Fukuoka, "Phase I Pharmacokinetic Trial of the Selective Oral Epidermal Growth Factor Receptor Tyrosine Kinase Inhibitor Gefitinib ('Iressa', ZD1839) in Japanese Patients with Solid Malignant Tumors,” Annals of Oncology, Vol. 14, No. 6, 2003, pp. 922-930. doi:10.1093/annonc/mdg250

[10] T. J. Lynch, D. W. Bell, R. Sordella, S. Gurubhagavatula, R. A. Okimoto, B. W. Brannigan, P. L. Harris, S. M. Haserlat, J. G. Supko, F. G. Haluska, D. N. Louis, D. C. Christiani, J. Settleman and D. A. Haber, "Activating Mutations in the Epidermal Growth Factor Receptor Underlying Responsiveness of Non-Small-Cell Lung Cancer to Gefitinib," The New England Journal of Medicine, Vol. 350, No. 21, 2004, pp. 2129-2139. doi:10.1056/NEJMoa040938

[11] J. G. Paez, P. A. Jänne, J. C. Lee, S. Tracy, H. Greulich, S. Gabriel, P. Herman, F. J. Kaye, N. Lindeman, T. J. Boggon, K. Naoki, H. Sasaki, Y. Fujii, M. J. Eck, W. R. Sellers, B. E. Johnson and M. Meyerson, "EGFR Mutations in Lung Cancer: Correlation with Clinical Response to Gefitinib Therapy,” Science, Vol. 304, No. 5676, 2004, pp. 1497-1500. doi:10.1126/science.1099314

[12] W. Pao, V. Miller, M. Zakowski, J. Doherty, K. Politi, I. Sarkaria, B. Singh, R. Heelan, V. Rusch, L. Fulton, E. Mardis, D. Kupfer, R. Wilson, M. Kris and H. Varmus, "EGF Receptor Gene Mutations Are Common in Lung Cancers from 'Never Smokers' and Are Associated with Sensitivity of Tumors to Gefitinib and Erlotinib," Proceedings of the National Academy of Sciences of the United States of America, Vol. 101, No. 36, 2004, pp. 13306-13311. doi:10.1073/pnas.0405220101

[13] M. L. Janmaat, F. A. Kruyt, J. A. Rodriguez and G. Giaccone, "Response to Epidermal Growth Factor Receptor Inhibitors in Non-Small Cell Lung Cancer Cells: Limited Antiproliferative Effects and Absence of Apoptosis Associated with Persistent Activity of Extracellular Signal-Regulated Kinase or Akt Kinase Pathways," Clinical Cancer Research, Vol. 9, No. 6, 2003, pp. 2316-2326.

[14] M. Ono, A. Hirata, T. Kometani, M. Miyagawa, S. Ueda, H. Kinoshita, T. Fujii and M. Kuwano, "Sensitivity to Gefitinib (Iressa, ZD1839) in Non-Small Cell Lung Cancer Cell Lines Correlates with Dependence on the Epidermal Growth Factor (EGF) Receptor/Extracellular Signal-Regulated Kinase 1/2 and EGF Receptor/Akt Pathway for Proliferation," Molecular Cancer Therapeutics, Vol. 3, No. 4, 2004, pp. 465-472.

[15] K. Takeuchi and F. Ito, "EGF Receptor in Relation to Tumor Development: Molecular Basis of Responsiveness of Cancer Cells to EGFR-Targeting Tyrosine Kinase Inhibitors," The FEBS Journal, Vol. 277, No .2, 2010, pp. 316-326.

[16] J. E. Chipuk, L. Bouchier-Hayes and D. R. Green, "Mitochondrial Outer Membrane Permeabilization during Apoptosis: The Innocent Bystander Scenario,” Cell Death and Differentiation, Vol. 13, No. 8, 2006, pp. 1396-1402. doi:10.1038/sj.cdd.4401963

[17] L. O’Connor, A. Strasser, L. A. O’Reilly, G. Hausmann, J. M. Adams, S. Cory and D. C. Huang, "Bim: A Novel Member of the Bcl-2 Family That Promotes Apoptosis," The EMBO Journal, Vol. 17, No. 2, 1998, pp. 384-395. doi:10.1093/emboj/17.2.384

[18] H. Puthalakath, D. C. Huang, L. A. O’Reilly, S. M. King and A. Strasser, "The Proapoptotic Activity of the Bcl-2 Family Member Bim Is Regulated by Interaction with the Dynein Motor Complex,” Molecular Cell, Vo. 3, No. 3, 1999, pp. 287-296. doi:10.1016/S1097-2765(00)80456-6

[19] M. Marani, T. Tenev, D. Hancock, J. Downward and N. R. Lemoine, "Identification of Novel Isoforms of the BH3 Domain Protein Bim Which Directly Activate Bax to Trigger Apoptosis,” Molecular and Cellular Biology, Vol. 22, No. 11, 2002, pp. 3577-3589. doi:10.1128/MCB.22.11.3577-3589.2002

[20] D. B. Costa, B. Halmos, A. Kumar, S. T. Schumer, M. S. Huberman, T. J. Boggon, D. G. Tenen and S. Kobayashi, "BIM Mediates EGFR Tyrosine Kinase Inhibitor-Induced Apoptosis in Lung Cancers with Oncogenic EGFR Mutations," PLoS Medicine, Vol. 4, No. 10, 2007, pp. 16691679. doi:10.1371/journal.pmed.0040315

[21] M. S. Cragg, J. Kuroda, H. Puthalakath, D. C. Huang and A. Strasser, "Gefitinib-Induced Killing of NSCLC Cell Lines Expressing Mutant EGFR Requires BIM and Can Be Enhanced by BH3 Mimetics,” PLoS Medicine, Vol. 4, No. 10, 2007, pp. 1681-1689.

doi:10.1371/journal.pmed.0040316

[22] Y. Gong, R. Somwar, K. Politi, M. Balak, J. Chmielecki, $\mathrm{X}$. Jiang and W. Pao, "Induction of BIM Is Essential for Apoptosis Triggered by EGFR Kinase Inhibitors in Mutant EGFR-Dependent Lung Adenocarcinomas," PLoS Medicine, Vol. 4, No. 10, 2007, pp. 1655-1668. doi:10.1371/journal.pmed.0040294

[23] R. Ley, K. Balmanno, K. Hadfield, C. Weston and S. J. Cook, "Activation of the ERK1/2 Signaling Pathway Promotes Phosphorylation and Proteasome-Dependent Degradation of the BH3-Only Protein, Bim,” Journal of Biological Chemistry, Vol. 278, No. 21, 2003, pp. 1881118816. doi:10.1074/jbc.M301010200

[24] K. Takeuchi, T. Shin-ya, K. Nishio and F. Ito, "MitogenActivated Protein Kinase Phosphatase-1 Modulated JNK Activation Is Critical for Apoptosis Induced by Inhibitor of Epidermal Growth Factor Receptor-Tyrosine Kinase," The FEBS Journal, Vol. 276, No. 5, 2009, pp. 1255-1265. doi:10.1111/j.1742-4658.2008.06861.x

[25] K. Takeuchi, Y. Motoda and F. Ito, "Role of Transcription Factor Activator Protein 1 (AP1) in Epidermal Growth Factor-Mediated Protection against Apoptosis Induced by a DNA-Damaging Agent,” The FEBS Journal, 
Vol. 273, No. 16, 2006, pp. 3743-3755.

doi:10.1111/j.1742-4658.2006.05377.x

[26] F. Luciano, A. Jacquel, P. Colosetti, M. Herrant, S. Cagnol, G. Pages and P. Auberger, "Phosphorylation of Bim-EL by Erk1/2 on Serine 69 Promotes Its Degradation via the Proteasome Pathway and Regulates Its Proapoptotic Function,” Oncogene, Vol. 22, No. 43, 2003, pp. 6785-6793. doi:10.1038/sj.onc.1206792

[27] R. Ley, K. E. Ewings, K. Hadfield and S. J. Cook, "Regulatory Phosphorylation of Bim: Sorting Out the ERK from the JNK," Cell Death and Differentiation, Vol. 12, No. 8, 2005, pp. 1008-1014. doi:10.1038/sj.cdd. 4401688

[28] P. F. Dijkers, R. H. Medema, J. W. Lammers, L. Koenderman and P. J. Coffer, "Expression of the Pro-Apoptotic Bcl-2 Family Member Bim Is Regulated by the Forkhead Transcription Factor FKHR-L1," Current Biology, Vol. 10, No. 19, 2000, pp. 1201-1204. doi:10.1016/S0960-9822(00)00728-4

[29] E. L. Greer and A. Brunet, "FOXO Transcription Factors at the Interface between Longevity and Tumor Suppression,” Oncogene, Vol. 24, No. 50, 2005, pp. 7410-7425. doi:10.1038/sj.onc.1209086

[30] Z. Fu and D. J. Tindall, "FOXOs, Cancer and Regulation of Apoptosis," Oncogene, Vol. 27, No. 16, 2008, pp. 2312-2319. doi:10.1038/onc.2008.24

[31] M. Stahl, P. F. Dijkers, G. J. Kops, S. M. Lens, P. J. Coffer, B. M. Burgering and R. H. Medema, "The Forkhead Transcription Factor FoxO Regulates Transcription of p27Kip1 and Bim in Response to IL-2,” Journal of Immunology, Vol. 168, No. 10, 2002, pp. 5024-5031.

[32] J. Gilley, P. J. Coffer and J. Ham, "FOXO Transcription Factors Directly Activate Bim Gene Expression and Promote Apoptosis in Sympathetic Neurons," Journal of Cell Biology, Vol. 162, No. 4, 2003, pp. 613-622. doi:10.1083/jcb.200303026

[33] A. Sunters, S. Fernández de Mattos, M. Stahl, J. J. Brosens, G. Zoumpoulidou, C. A. Saunders, P. J. Coffer, R. H. Medema, R. C. Coombes and E. W. Lam, "FoxO3a Transcriptional Regulation of Bim Controls Apoptosis in Paclitaxel-Treated Breast Cancer Cell Lines,” The Journal of Biological Chemistry, Vol. 278, No. 50, 2003, pp. 49795-49805. doi:10.1074/jbc.M309523200

[34] A. Essafi, S. Fernández de Mattos, Y. A. Hassen, I. Soeiro, G. J. Mufti, N. S. Thomas, R. H. Medema and E. W. Lam, "Direct Transcriptional Regulation of Bim by FoxO3a Mediates STI571-Induced Apoptosis in BcrAbl-Expressing Cells,” Oncogene, Vol. 24, No. 14, 2005, pp. 2317-2329. doi:10.1038/sj.onc.1208421

[35] R. Ley, K. E. Ewings, K. Hadfield, E. Howes, K. Balmanno and S. J. Cook, "Extracellular Signal-Regulated Kinases 1/2 Are Serum-Stimulated 'Bim(EL) Kinases' That Bind to the BH3-Only Protein Bim(EL) Causing Its Phosphorylation and Turnover," The Journal of Biological Chemistry, Vol. 279, No. 10, 2004, pp. 8837-8847. doi:10.1074/jbc.M311578200

[36] R. Ley, K. Hadfield, E. Howes and S. J. Cook, "Identification of a DEF-Type Docking Domain for Extracellular
Signal-Regulated Kinases 1/2 That Directs Phosphorylation and Turnover of the BH3-Only Protein BimEL," The Journal of Biological Chemistry, Vol. 280, No. 18, 2005, pp. 17657-17663. doi:10.1074/jbc.M412342200

[37] N. Galili, R. J. Davis, W. J. Fredericks, S. Mukhopadhyay, F. J. Rauscher III, B. S. Emanuel, G. Rovera and F. G. Barr, "Fusion of a Fork Head Domain Gene to PAX3 in the Solid Tumour Alveolar Rhabdomyosarcoma," Nature Genetics, Vol. 5, No. 3, 1993, pp. 230-235. doi:10.1038/ng1193-230

[38] M. J. Anderson, C. S. Viars, S. Czekay, W. K. Cavenee and K. C. Arden, "Cloning and Characterization of Three Human Forkhead Genes That Comprise an FKHR-Like Gene Subfamily,” Genomics, Vol. 47, No. 2, 1998, pp. 187-199. doi:10.1006/geno.1997.5122

[39] J. Hillion, M. Le Coniat, P. Jonveaux, R. Berger and O. A. Bernard, "AF6q21, a Novel Partner of the MLL Gene in t(6;11)(q21;q23), Defines a Forkhead Transcriptional Factor Subfamily,” Blood, Vol. 90, No. 9, 1997, pp. 37143719.

[40] A. Borkhardt, R. Repp, O. A. Haas, T. Leis, J. Harbott, J. Kreuder, J. Hammermann, T. Henn and F. Lampert, "Cloning and Characterization of AFX, the Gene That Fuses to MLL in Acute Leukemias with a t(X;11)(q13;q23)," Oncogene, Vol. 14, No. 2, 1997, pp. 195-202.

[41] A. Brunet, A. Bonni, M. J. Zigmond, M. Z. Lin, P. Juo, L. S. Hu, M. J. Anderson, K. C. Arden, J. Blenis and M. E. Greenberg, "Akt Promotes Cell Survival by Phosphorylating and Inhibiting a Forkhead Transcription Factor," Cell, Vol. 96, No. 6, 1999, pp. 857-868. doi:10.1016/S0092-8674(00)80595-4

[42] D. R. Plas and C. B. Thompson, "Akt Activation Promotes Degradation of Tuberin and FOXO3a via the Proteasome," The Journal of Biological Chemistry, Vol. 278, No. 14, 2003, pp. 12361-12366. doi:10.1074/jbc.M213069200

[43] J. Y. Yang, C. S. Zong, W. Xia, H. Yamaguchi, Q. Ding, X. Xie, J. Y. Lang, C. C. Lai, C. J. Chang, W. C. Huang, H. Huang, H. P. Kuo, D. F. Lee, L. Y. Li, H. C. Lien, X. Cheng, K. J. Chang, C. D. Hsiao, F. J. Tsai, C. H. Tsai, A. A. Sahin, W. J. Muller, G. B. Mills, D. Yu, G. N. Hortobagyi and M. C. Hung, "ERK Promotes Tumorigenesis by Inhibiting FOXO3a via MDM2-Mediated Degradation,” Nature Cell Biology, Vol. 10, No. 2, 2008, pp. 138148. doi:10.1038/ncb1676

[44] M. C. Hu, D. F. Lee, W. Xia, L. S. Golfman, F. Ou-Yang, J. Y. Yang, Y. Zou, S. Bao, N. Hanada, H. Saso, R. Kobayashi and M. C. Hung, "IkappaB Kinase Promotes Tumorigenesis through Inhibition of Forkhead FOXO3a," Cell, Vol. 117, No. 2, 2004, pp. 225-237. doi:10.1016/S0092-8674(04)00302-2

[45] A. Brunet, F. Kanai, J. Stehn, J. Xu, D. Sarbassova, J. V. Frangioni, S. N. Dalal, J. A. DeCaprio, M. E. Greenberg and M. B. Yaffe, "14-3-3 Transits to the Nucleus and Participates in Dynamic Nucleocytoplasmic Transport,” The Journal of Cell Biology, Vol. 156, No. 5, 2002, pp. 817828. doi:10.1083/jcb.200112059

[46] L. P. Van Der Heide, M. F. Hoekman and M. P. Smidt, 
"The Ins and Outs of FoxO Shuttling: Mechanisms of FoxO Translocation and Transcriptional Regulation," The Biochemical Journal, Vol. 380, No. 2, 2004, pp. 297-309. doi:10.1042/BJ20040167

[47] X. Wang, W. R. Chen and D. Xing, “A Pathway from JNK through Decreased ERK and Akt Activities for FOXO3a Nuclear Translocation in Response to UV Irradiation,” Journal of Cellular Physiology, Vol. 227, No. 3, 2012, pp. 1168-1178. doi:10.1002/jcp.22839

[48] A. Sunters, P. A. Madureira, K. M. Pomeranz, M. Aubert, J. J. Brosens, S. J. Cook, B. M. Burgering, R. C. Coombes and E. W. Lam, "Paclitaxel-Induced Nuclear Translocation of FOXO3a in Breast Cancer Cells Is Mediated by c-Jun NH2-Terminal Kinase and Akt," Cancer Research, Vol. 66, No. 1, 2006, pp. 212-220. doi:10.1158/0008-5472.CAN-05-1997

[49] M. A. Essers, S. Weijzen, A. M. de Vries-Smits, I. Saarloos, N. D. de Ruiter, J. L. Bos and B. M. Burgering, "FOXO Transcription Factor Activation by Oxidative Stress Mediated by the Small GTPase Ral and JNK," The EMBO Journal, Vol. 23, No. 24, 2004, pp. 4802-4812. doi:10.1038/sj.emboj.7600476

[50] M. Potente, C. Urbich, K. Sasaki, W. K. Hofmann, C. Heeschen, A. Aicher, R. Kollipara, R. A. DePinho, A. M. Zeiher and S. Dimmeler, "Involvement of Foxo Transcription Factors in Angiogenesis and Postnatal Neovascularization," The Journal of Clinical Investigation, Vol. 115, No. 9, 2005, pp. 2382-2392. doi:10.1172/JCI23126

[51] K. Okamoto, I. Okamoto, W. Okamoto, K. Tanaka, K. Takezawa, K. Kuwata, H. Yamaguchi, K. Nishio and K.
Nakagawa, "Role of Survivin in EGFR Inhibitor-Induced Apoptosis in Non-Small Cell Lung Cancers Positive for EGFR Mutations," Cancer Research, Vol. 70, No. 24, 2010, pp. 10402-10410. doi:10.1158/0008-5472.CAN-10-2438

[52] W. Pao, V. A. Miller, K. A. Politi, G. J. Riely, R. Somwar, M. F. Zakowski, M. G. Kris and H. Varmus, "Acquired Resistance of Lung Adenocarcinomas to Gefitinib or Erlotinib Is Associated with a Second Mutation in the EGFR Kinase Domain,” PLoS Medicine, Vol. 2, No. 3, 2005, pp. 225-235. doi:10.1371/journal.pmed.0020073

[53] J. Bean, C. Brennan, J. Y. Shih, G. Riely, A. Viale, L. Wang, D. Chitale, N. Motoi, J. Szoke, S. Broderick, M. Balak, W. C. Chang, C. J. Yu, A. Gazdar, H. Pass, V. Rusch, W. Gerald, S. F. Huang, P. C. Yang, V. Miller, M. Ladanyi, C. H. Yang and W. Pao, "MET Amplification Occurs with or without T790M Mutations in EGFR Mutant Lung Tumors with Acquired Resistance to Gefitinib or Erlotinib," Proceedings of the National Academy of Sciences of the United States of America, Vol. 104, No. 5, 2007, pp. 20932-20937. doi:10.1073/pnas.0710370104

[54] J. A. Engelman, K. Zejnullahu, T. Mitsudomi, Y. Song, C. Hyland, J. O. Park, N. Lindeman, C. M. Gale, X. Zhao, J. Christensen, T. Kosaka, A. J. Holmes, A. M. Rogers, F. Cappuzzo, T. Mok, C. Lee, B. E. Johnson, L. C. Cantley and P. A. Jänne, "MET Amplification Leads to Gefitinib Resistance in Lung Cancer by Activating ERBB3 Signaling,” Science, Vol. 316, No. 5827, 2007, pp. 1039-1043. doi:10.1126/science.1141478

ERK: extracellular signal-regulated kinase;

MKP-1: mitogen-activated protein kinase phosphatase-1;

NSCLC: non-small-cell lung cancer;

FOXO: forkhead box proteins $\mathrm{O}$. 